éxito del diagnóstico de esta enfermedad en países como Costa Rica se favorece por la diversidad de especies hospedadoras. Muchos estudios identificaron la presencia de esta bacteria en ganado vacuno, cerdos, caballos, cabras, ovejas, perros y roedores. ${ }^{7,8}$ Además, se deben considerar otros factores de riesgo para la adquisición de la leptospirosis. En la actualidad hay un incremento en las actividades recreativas en ríos y lagos, lo que incrementa el riesgo para adquirir la leptospirosis. Este riesgo se explica por la contaminación de fuentes de agua con orina de animales infectados, factor relacionado con la ocurrencia de brotes de leptospirosis. ${ }^{9}$

\section{Agradecimientos}

A la Licda. Marlen Solís Mata, al Dr. Luis González Salas, por sus valiosas sugerencias. También al técnico de Microbiología Juan Israel Chaverri Salazar, por su apreciable colaboración.

\section{Referencias}

1. Stefos A, Georgiadou SP, Gioti C, Loukopoulos A, Ioannou M, Pournaras S, et al. Leptospirosis and pancytopenia: two case reports and review of the literature. J Infect. 2005; 51: 277-80.
2. Erdinc FS, Koruk ST, Hatipoglu CA, Kinikli S, Demiroz AP. Three cases of anicteric leptospirosis from Turkey: Mild to severe complications. J Infect. 2006; 52:45-48.

3. Farrar We. Leptospira species (leptospirosis). In Mandell GL, Bennett JE, Dolin R, editors. Principles and practice of infectious diseases. 4th ed. New York: Churchill Livingstone; 1995. p2137-41.

4. Feigin RD, Anderson DC. Human leptospirosis. Crit Rev Clin Lab Sci. 1975; 5:413-67.

5. Flannery B, Pereira M, Freitas L, Carvalho C, Góes L, De Saboia G, et al. Referal pattern of leptospirosis cases during a large urban epidemic of dengue. Am J Trop Med Hyg. 2001; 65:657-63.

6. Bruce M, Sanders E, Leake J, Zaidel O, Bragg S, Aye T, et al. Leptospirosis among patients presenting with dengue-like illness in Puerto Rico. Acta Trop. 2005; 96:36-46.

7. Homem V, Heinemann M, Moraes Z, Vasconcellos S, Ferreira F, Ferreira N. Estudo epidemiológico da leptospirose bovina e humana na Amazônia oriental brasileira. Rev Soc Bras Med Trop. 2001; 34:173-80.

8. Vado I, Cardenas M, Jiménez B, Alzina A, Laviada H, Suarez V, et al. Clinical-epidemiological study of leptospirosis in humans and reservoirs in Yucatán, Mexico. Rev Inst Med Trop S Paulo. 2002; 44:335-40

9. Katz A, Ansdell V, Effler P, Middleton C, Sasaki D. Leptospirosis in Hawaii, 1974-1998: epidemiologic analysis of 353 laboratoryconfirmed cases. Am J Trop Med Hyg. 2002; 66:61-70.

\title{
Mielolipoma, una lesión quirúrgica poco frecuente de la glándula adrenal
}

\section{Myelolipoma, an infrequent surgical lesion of the adrenal gland}

\author{
Humberto Álvarez-Pertuz, Laura Arroyo-Martínez, Jorge Acuña-Calvo
}

\section{Resumen}

El mielolipoma es un tumor benigno poco frecuente, compuesto de tejido adiposo maduro y elementos hematopoyéticos diversos, histológicamente similares a la médula ósea normal. Estos tumores se pueden localizar comúnmente en las glándulas adrenales, pero también en sitios extra adrenales. Puede encontrarse como tumor único o múltiple, y es hormonalmente inactivo. ${ }^{1}$ El origen del mielolipoma es desconocido y se han propuesto varias teorías. Los mielolipomas muestran una gran variedad de manifestaciones clínicas, tales como: sangrado digestivo, hematuria, dolor abdominal en los flancos, masa abdominal y pérdida de peso, entre otras. La incidencia de estos tumores

Servicio de Cirugía General, Hospital San Rafael de Alajuela, Caja Costarricense de Seguro Social.

Abreviaturas: $B A A F$, biopsia por aspiración con aguja fina; $H C D$, hipocondrio derecho; MLA, mielolipoma adrenal; RM, resonancia magnética; SDA, sangrado digestivo alto SDB, sangrado digestivo bajo;TAC, tomografía axial computarizada; US, ultrasonido.

ISSN 0001-6002/2008/50/1/54-57

Acta Médica Costarricense, (C2008

Colegio de Médicos y Cirujanos en autopsias es del 0,08 al 0,2\%, gracias al US, la TAC y la $\mathrm{RM}$, su hallazgo es mas frecuente hoy. ${ }^{2}$ El diagnóstico es generalmente por exclusión, habiéndose descartado otras entidades suprarrenales. Entre los métodos diagnósticos figuran: la TAC, el US y la RM. La resección quirúrgica es el tratamiento de elección, cuando haya sintomatología; de lo contrario, un manejo conservador con controles periódicos del paciente será más que suficiente. ${ }^{3}$ Se presenta el caso de una paciente de 55 años, hipertensa, obesa y con un trastorno depresivo-ansioso, quien consultó por dolor abdominal, náuseas y vómitos.

\section{Abstract}

Myelolipoma is a non functioning benign tumor of the adrenal gland which is formed by hematopoietic and adipose tissue. The tumor was first described by Gierke in 1905, and named by Oberling in 1929. Most series consist of myelolipomas discovered incidentally at autopsy. They are generally located in the adrenal gland, although they can occur in other organs. 
Endocrine and/or metabolic disorders associated with AML include obesity, Type II Diabetes, congenital adrenal hyperplasia due to 21 or 17 alpha-hydroxylase deficiency, Cushing's disease, Conn's syndrome, pheochromocytoma and polycystic ovarian disease.

Myelolipomas are asymptomatic, but they may cause pain secondary to bleeding, hematuria or mass; when symptomatic, the most consistent complaint is abdominal pain caused by hemorrhage within the tumor. Ultrasound combined with computed tomography is useful in diagnosis. Definitive diagnosis and treatment are accomplished by simple excision and histological report; radical surgery is unnecessary.

We present here the case of a 55 years old female who came in with abdominal pain, nausea and vomiting. After an abdominal ultrasound, an abdominal CT scan and eventually, surgical removal, an adrenal myelolipoma was found. She did well after surgery and remains asymptomatic.

Descriptores: mielolipoma, sangrado digestivo, glándula adrenal, tumor benigno, tejido hematopoyético, laparotomía exploratoria

Key words: myelolipoma, G.I bleeding, adrenal gland, bening tumor, hematopoietic tissue, exploratory laparotomy.

Recibido: 19 de febrero de 2007

Aceptado: 12 de junio de 2007

\section{Caso clínico}

Femenina de 55 años, obesa, hipertensa en tratamiento con atenolol $100 \mathrm{mg}$ por día PO, hidroclorotiazida $25 \mathrm{mg}$ por día PO, y trastorno depresivo - ansioso en tratamiento con fluoxetina $25 \mathrm{mg}$ por día PO, y Lorazepam 1 tableta por noche PO. Consultó al Servicio de Emergencias del Hospital San Rafael de Alajuela, por dolor abdominal en hipocondrio derecho, intenso, tipo cólico, irradiado a la espalda; asociaba náuseas y vómitos.

A la exploración física se encontró dolor a la palpación en HCD con signo de Murphy +. Un US de abdomen general demostró un hígado de tamaño normal, sin masas, vesícula distendida con un lito de $15 \mathrm{~mm}$ en su interior, vía biliar de calibre normal y, como hallazgo incidental, en el polo superior del riñón derecho, se identificó una masa ecogénica de 8.8 × $9.3 \times 7.6 \mathrm{~cm}$, compatible con una lesión de la glándula adrenal derecha.

Se realizó una TAC de abdomen con doble medio de contraste; se demostró en el polo superior del riñón derecho, una masa sólida con densidades negativas que sugieren contenido graso, con contornos bien definidos, que "podría corresponder a un angiomielolipoma renal, dada a la densidad y el realce con el medio de contraste".
La arteriografía renal no identificó vascularidad propia de dicha lesión, los exámenes de laboratorio preoperatorios, incluyendo determinaciones hormonales en orina de 24 horas, fueron normales.

Se le realizó una laparotomía exploratoria, colecistectomía y exploración retroperitoneal derecha con resección completa de la lesión adrenal derecha, ovalada, la cual se encontró bien delimitada, encapsulada y de consistencia blanda. La superficie de esta presentaba una cápsula de tejido fibroso.

La paciente evolucionó satisfactoriamente, fue egresada en el segundo día de postoperatorio y el informe anatomopatológico demostró células de la corteza suprarrenal, mezcladas con numerosas células de corte hematopoyético y grasa, diagnóstico de un MLA. Actualmente, lleva su control satisfactorio, en Consulta Externa de Cirugía General.

\section{Discusión}

En 1905, Gierke describió por primera vez este tumor, pero el término "mielolipoma" fue acuñado por Oberling en 1929. Hasta 1957, todas las lesiones descritas fueron hallazgos incidentales en autopsias, por lo que se les llamaban incidentalomas; en ese mismo año, Dyckman y Freedman reportaron el primer caso de MLA sintomático de la historia, el cual se resecó quirúrgicamente de manera satisfactoria. ${ }^{4}$

El MLA es un tumor benigno raro, originado de la corteza adrenal, compuesto por una cantidad variable de tejido adiposo maduro y elementos hematopoyéticos de las 3 series y en cualquier estadio. Su superficie consiste de una pseudocápsula comprimida de zona glomerular y fascicular.

Por lo general, son asintomáticos, unilaterales, de tamaño variable. La mayoría de los autores señalan que miden de $3 \mathrm{a} 10 \mathrm{~cm}$, pero se han encontrado algunos gigantes. Los hay desde muy pequeños y asintomáticos, hasta aquellos que son clínicamente palpables, sintomáticos y de más de 30 $\mathrm{cm}$. Se citan en la bibliografía mielolipomas mayores de 5.900 gramos. $^{5}$

La frecuencia en autopsias oscila entre el $0,08 \%$ y el 0,8 $\%$. Puede haber necrosis grasa y hemorragia, comúnmente en aquellos tumores sintomáticos.

Soós ha descrito macroscópicamente dos tipos de MLA: Tipo I: anaranjado -amarillento, predominantemente lipomatoso, asociado con una mínima cantidad de elementos hematopoyéticos, mayormente eritroides; Tipo II: parduzco, con elementos de predominio mieloide. ${ }^{6}$

Teorías acerca de la etiología de este tumor incluyen: hematopoyesis extramedular, embolismo de células de la médula ósea y metaplasia de células corticales. La teoría que hoy tiene mayor aceptación es la que sugiere que las células 
de la mesénquima indiferenciadas dentro de la corteza adrenal son estimuladas para diferenciarse hacia la línea mieloide o lipoide, pero el estímulo aún se desconoce.

Los MLA ocurren predominantemente en el sexo masculino comparado con el femenino, en una relación de 2-3, en los caucásicos. Es esencialmente una entidad de los adultos, que ocurre entre la quinta y séptima década de vida. Involucra más frecuentemente la glándula adrenal derecha que la izquierda (1-3), el compromiso bilateral es muy raro. No se ha reportado ninguna muerte ni tampoco transformación maligna. Se pueden complicar con ruptura y hemorragia.

Algunos trastornos endocrinos o metabólicos están asociados a este tipo de tumores, entre ellos: "diabetes mellitus" tipo II, obesidad, hipertensión arterial, hiperplasia congénita adrenal por deficiencia de la 21 o 17 alphahidroxilasa, Síndrome de Cushing, feocromocitoma y síndrome de ovarios poliquísticos.

La mayoría ocurren en la glándula suprarrenal $(85 \%)$, usualmente tienen nidos de células adrenales y carecen de cápsula verdadera que los separe de ella. Histológicamente, la llamada pseudocápsula consta de restos de corteza y la cápsula adrenal.

Con menor frecuencia, se describen los extra adrenales, que representan un $15 \%$ y se ubican en: el retroperitoneo, la región presacral, renal, perirenal, en el mediastino, la pleura, el hígado, el estómago, las fosas iliacas y la fascia muscular. No contienen tejido adrenal, son encapsulados y bien circunscritos (Cuadro 1).

El MLA es usualmente asintomático, pero en ocasiones se presenta con dolor en los flancos, el cual puede ser secundario a hemorragia intratumoral o peritumoral, hematuria o la masa. Se han asociado otras manifestaciones como hepatomegalia o sangrado digestivo alto o bajo, y se han hallado entidades asociadas como carcinoma no metastásico, adenoma hipofisiario, hidronefrosis, pielonefritis, anemia perniciosa o megaloblástica. La presentación más dramática del mielolipoma es el sangrado agudo (Cuadro 2).

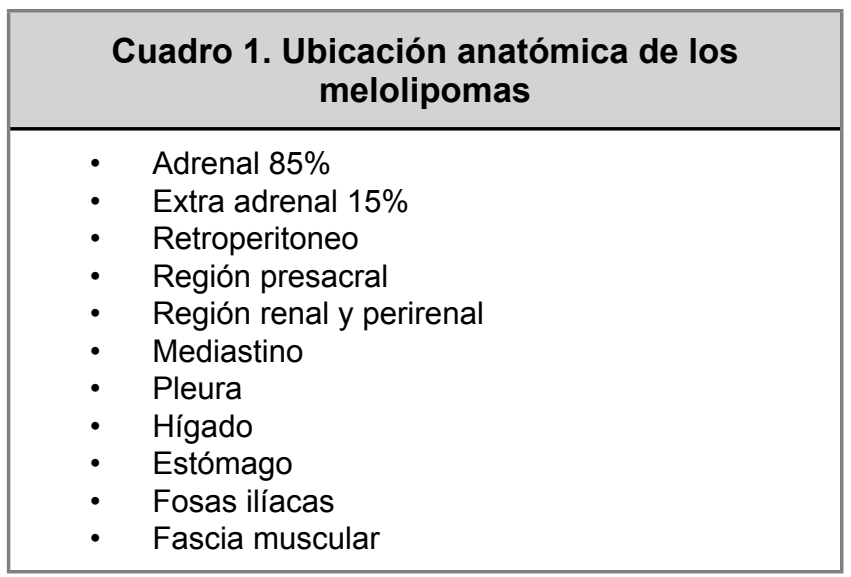

El diagnóstico generalmente se hace por exclusión una vez que se han descartado otras alteraciones adrenales. Entre los métodos diagnósticos figuran: el US, la TAC, la RM, y la cintigrafía con 1311-6-beta-iodometil-19-norcolesterol. El método de imagen más preciso para diagnóstico es la TAC, que reconoce lesiones muy pequeñas $(<1 \mathrm{~cm})$ y permite diferenciar entre mielolipoma, hematoma agudo o crónico, quiste o hiperplasia.

Topográficamente, el MLA es una masa bien definida, con cápsula de densidad grasa y con atenuación de $-30 \mathrm{a}$ 115 unidades hounsfield. La biopsia por aspiración con aguja fina es otra modalidad que permite confirmar el diagnóstico hecho con medios de imagen, la cual no solo es capaz de diagnosticar el mielolipoma, sino también otras anormalidades suprarrenales.

El diagnóstico diferencial incluye: adenoma, carcinoma, angiomielolipomarenal, liposarcomaylinfomaretroperitoneal, carcinoma renal y hematopoyesis extramedular.

El consenso general, según la bibliografía, es que el tumor se debe operar cuando está complicado, ya sea por efecto de masa, dolor, sangrado, ruptura o sepsis urinaria a repetición. En relación con el tamaño del tumor, no existe un acuerdo general de cuál sea la medida óptima para decidir el manejo quirúrgico.

El manejo del MLA debe ser individualizado, a los pacientes con tumores $<4 \mathrm{~cm}$ de diámetro y asintomáticos, diagnosticados por imágenes o histología, es mejor realizarles revisiones periódicas. La resección quirúrgica es la opción por seguir en aquellos tumores grandes y sintomáticos que exhiben zonas de hemorragia o necrosis, o en aquellos donde se sospecha malignidad. ${ }^{7}$

Cuando a un paciente con MLA se le brinda manejo expectante, es necesario tener un diagnóstico preciso de la lesión y excluir tumores malignos. Se deben hacer estudios hormonales y diagnosticar el mielolipoma con medios radiológicos, apoyados con BAAF. ${ }^{8}$

Se recomiendan revisiones periódicas a los pacientes, con una TAC de abdomen de control cada 3, 6, 12 y 18 meses luego del diagnóstico inicial, para llevar un control estrecho de la lesión y su evolución. ${ }^{9}$

\section{Agradecimientos}

A los Drs. Grace Masís, Álvaro Duval y Mauricio Segura, por su invaluable colaboración.

Cuadro 2. Manifestaciones clínicas de los MLA

- Asintomático

- Dolor en flancos

- Hematuria

- SDA/SDB

- Hepatomegalia

- Masa palpable 


\section{Referencias}

1. Silva, Ortíz, José, Dr., Virgilio, Hernández, Rubén, Dr., Fonseca, Morales, José, Dr., Belmonte, Montes, Carlos, Dr., Magaña, Sánchez, Ignacio, Dr., Cough, Molina, Jorge, Msc. Mielolipoma adrenal: informe de dos pacientes. Cir Gen 2000; 22: 242-246.

2. Terry, M, Mónica, Ochoa, V, Carlos, Tamayo, C, Nahum, Siu Ting, Ch, Gloria, Terry, Eduardo, Palomino, D, Victoria. Mielolipoma adrenal: Reporte de un caso. Rev Soc Peruana Med Int 2003; 16: 107110 .

3. Han, M, Burnett, A, L, Fishman, E, K, Marshall, F, F. The Natural history and treatment of adrenal myelolipoma. J Urol 1997; 157: 1213-1216.

4. Filobbos, S, A, Seddon. J. A. Myelolipoma of the adrenal. Br J Surg 1980; 67:147-148.

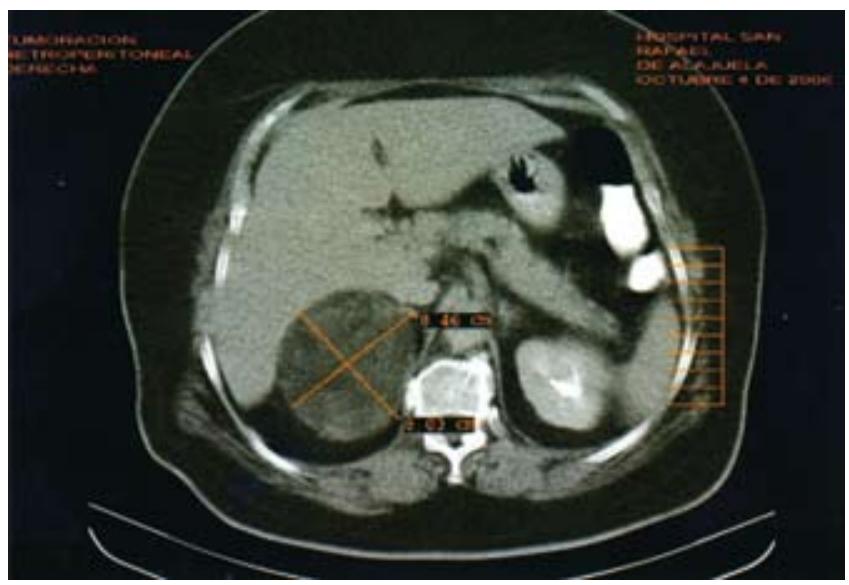

Figura 1. TAC de abdomen: evidencia masa sólida en el polo superior del riñón derecho, de 8,46 × 8,03 cm; de densidad grasa y contornos bien definidos.
5. Goldman, H, B, Howard, R, C, Patterson, A,L. Spontaneous retroperitoneal hemorrhage from a giant adrenal myelolipoma. J Urol 1996; 115: 639-640.

6. Pagana, J, Timothy, MD., Karasick, J, Steven, MD., Karasick, David, MD., Stahlgren, Leroy, MD. Myelolipoma of the adrenal gland. Am J Sur 1981; 141: 282-285.

7 A, Bhansali, RJ, Dash, SK, Singh, A, Behra, P, Singh, BD, Radotra. Adrenal Myelolipoma: profile of six patients with a brief review of literature. Int J Endocrinol Metab 2003; 1: 33-40.

8 Han, M, Burnett, A, L, Fishman, E, K, Marshall, F, F. The Natural history and treatment of adrenal myelolipoma. J Urol 1997; 157 : 1213-1216

9 Noble, J, Mark, MD., Montague, Drogo, K, MD., Levin, Howard, S, MD. Myelolipoma: an unusual surgical lesion of the adrenal gland. Cancer. 1982; 49: 952-958.

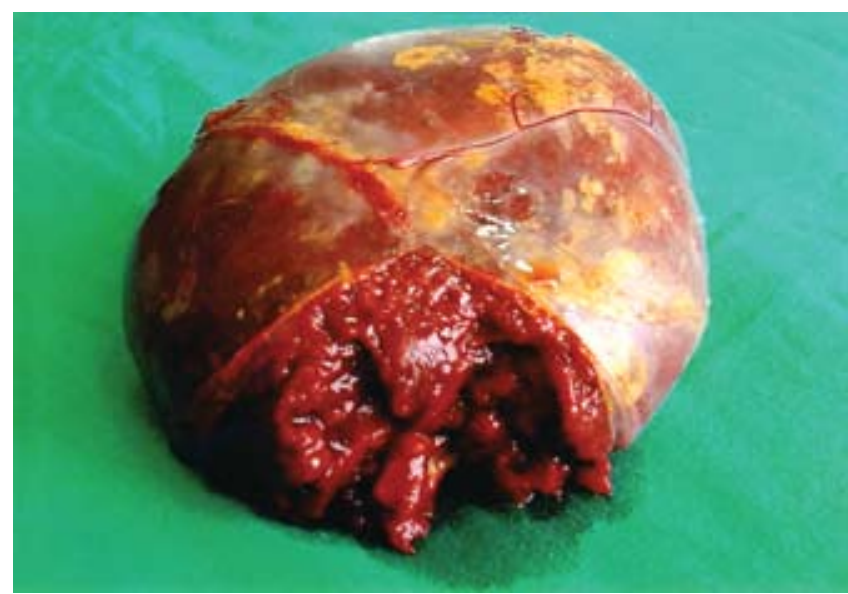

Figura 2. Masa oval de $10 \times 8,0 \times 4,5 \mathrm{~cm}$ de consistencia blanda que pesó 230 gramos. La superficie externa presenta una fina cápsula de tejido fibroso. Al corte se observa una masa grasa que compromete toda la región central que contrasta con extensas áreas hemorrágicas. Estudio histopatológico compatible con mielolipoma adrenal

\title{
Insuficiencia renal aguda asociada a picadura de abeja africanizada
}

\section{Acute renal failure secondary to africanized bee stings}

\author{
Verny Huertas-Franco, Jorge Bucknor-Masís
}

\section{Resumen}

Se presenta el caso de un adulto mayor diabético tipo 2, que sufrió un ataque masivo de abejas africanizadas, y llegó a la sala de emergencias tres horas después del inicio del ataque. El paciente presentó insuficiencia renal aguda por combinación de factores, y se recuperó con tratamiento de diálisis temprana. Se discuten los problemas que refieren estos pacientes, y cuál sería el manejo ideal de tales casos.

\section{Sección de Medicina, Hospital San Juan de Dios}

Correspondencia: Verny Huertas Franco, Apdo. 125-1002 Paseo de los Estudiantes, San José, Costa Rica

ISSN 0001-6002/2008/50/1/57-60

Acta Médica Costarricense, (C2008

Colegio de Médicos y Cirujanos
Descriptores: Abeja africanizada, abeja africana, insuficiencia renal aguda, diálisis peritoneal, hemodiálisis.

\section{Abstract \\ This is the case of an elderly patient who suffered multiple Africanized bee stings. He developed acute renal failure as a complication. He however recovered completely after treatment with peritoneal dialysis and hemodialysis.}

Key words: Africanized bee, african bee, acute renal failure, peritoneal dialysis, hemodialysis

Recibido: 23 de abril de 2007

Aceptado: 4 de septiembre de 2007 